\title{
ISGylation in Innate Antiviral Immunity and Pathogen Defense Responses: A Review
}

\author{
Mengdi Zhang ${ }^{1}$, Jingxian $L i^{2}$, Haiyan Yan ${ }^{1}$, Jun Huang ${ }^{2}$, Fangwei Wang ${ }^{2}$, Ting Liu ${ }^{2}$, \\ Linghui Zeng ${ }^{1 *}$ and Fangfang Zhou ${ }^{3 *}$
}

\begin{abstract}
${ }^{1}$ School of Medicine, Zhejiang University City College, Hangzhou, China, ${ }^{2}$ MOE Laboratory of Biosystems Homeostasis and Protection and Innovation Center for Cell Signaling Network, Life Sciences Institute, Zhejiang University, Hangzhou, China,

${ }^{3}$ Institute of Biology and Medical Sciences, Soochow University, Suzhou, China
\end{abstract}

OPEN ACCESS

Edited by:

Ana Cuenda,

Consejo Superior de Investigaciones

Cientificas (CSIC), Spain

Reviewed by:

Zhenghong Lin,

Chongqing University, China

Zhongwei Li,

University of Southern California, Los

Angeles, United States

*Correspondence:

Fangfang Zhou

zhoufangfang@suda.edu.cn

Linghui Zeng

zenglh@zucc.edu.cn

Specialty section:

This article was submitted to

Signaling,

a section of the journal

Frontiers in Cell and Developmental

Biology

Received: 02 October 2021

Accepted: 22 October 2021

Published: 25 November 2021

Citation:

Zhang M, Li J, Yan H, Huang J, Wang F, Liu T, Zeng L and Zhou F (2021) ISGylation in Innate Antiviral

Immunity and Pathogen Defense

Responses: A Review.

Front. Cell Dev. Biol. 9:788410.

doi: 10.3389/fcell.2021.788410
The interferon-stimulating gene 15 (ISG15) protein is a ubiquitin-like protein induced by interferons or pathogens. ISG15 can exist in free form or covalently bind to the target protein through an enzymatic cascade reaction, which is called ISGylation. ISGylation has been found to play an important role in the innate immune responses induced by type I interferon, and is, thus, critical for the defense of host cells against RNA, DNA, and retroviruses. Through covalent binding with the host and viral target proteins, ISG15 inhibits the release of viral particles, hinder viral replication, and regulates the incubation period of viruses, thereby exerting strong antiviral effects. The SARS-CoV-2 papain-like protease, a virus-encoded deubiquitinating enzyme, has demonstrated activity on both ubiquitin and ISG15 chain conjugations, thus playing a suppressive role against the host antiviral innate immune response. Here we review the recent research progress in understanding ISG15-type ubiquitin-like modifications, with an emphasis on the underlying molecular mechanisms. We provide comprehensive references for further studies on the role of ISG15 in antiviral immunity, which may enable development of new antiviral drugs.

Keywords: ISG15, isgylation, immune response, innate antiviral immunity, SARS PLpro

\section{INTRODUCTION}

Interferon-stimulated gene 15 (ISG15) is a member of the family of interferon-stimulating genes (ISGs) (Takeuchi et al., 2019), which are fast and strong type I interferon (IFN)-stimulated reaction proteins that inhibit viral replication, whose function against virus invasion has been fully investigated (Loeb and Haas, 1992; Hermann and Bogunovic, 2017; Sooryanarain et al., 2017). Viral infection induces IFN synthesis, and the secreted IFN acts on nearby uninfected cells to resist the infection. After the virus enters the body, IFN binds to IFN receptors, which activate the Janus protein tyrosine kinase-signal transducer and activator of transcription pathway to form the interferon-stimulating factor 3 complex, which induces the expression of hundreds of ISGs, including ISG15, which can fight against the replication and invasion of the virus (Yuan and Krug, 2001).

Recently, the function of ISG15 as a ubiquitin-like protein has attracted much attention. ISG15 is the first identified ubiquitin-like protein, which contains two ubiquitin-like domains, and its amino acid sequence shows 50\% homology with ubiquitin (Dos Santos and Mansur, 2017). Under physiological conditions, the ISG15 precursor protein can be cleaved into a mature $15-\mathrm{kDa}$ form, exposing the carboxyl-terminated LRLRGG motif, which recognizes and binds to substrate 


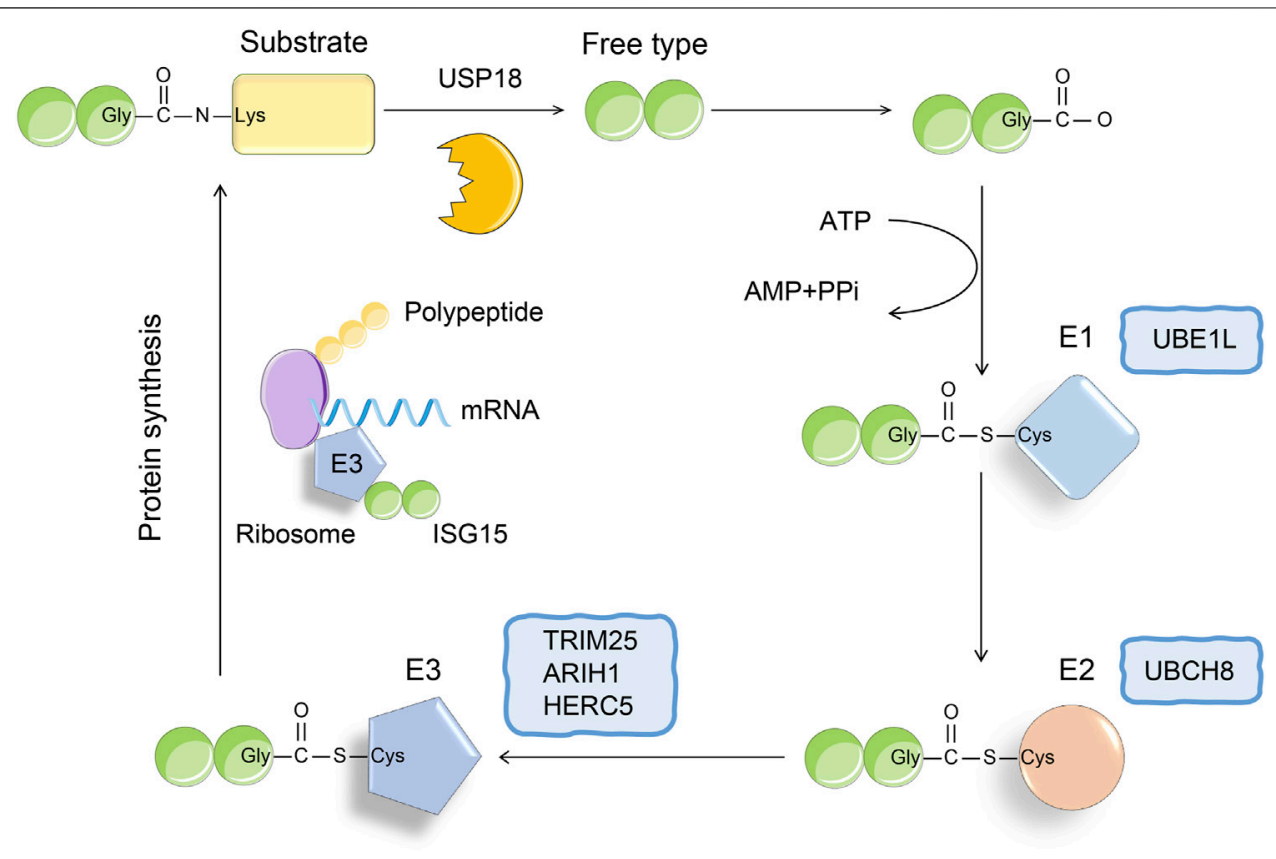

FIGURE 1 | The conjugation of ISG15. The binding process of ISG15 and substrate is similar to the three-step enzyme cascade reaction of ubiquitination. The formation of thioester bond between E1 activating enzyme (UBE1L) and ISG15 depends on ATP, thereby activating ISG15. Next, SIG15 is transferred to the cysteine active site of E2 ligase (UBCH8). Finally, E3 ligase binds to polysomes, thereby promoting the binding of ISG15 to the nascent target protein. The process of ISGylation is reversible, and USP18 as a deubiquitinating enzyme can specifically remove ISG15 from the binding protein.

lysine residues, resulting in its ISGylation (Figure 1) (Potter et al., 1999; Langevin et al., 2013; Zuo et al., 2016). Similar to ubiquitin modification, ISG modification of the substrate is also catalyzed by ubiquitin-activating enzyme E1, ubiquitin-binding enzyme E2, and ubiquitin ligase E3 (Mustachio et al., 2018). The removal of substrate ISGylation is catalyzed by deubiquitinases (DUB), and ubiquitin-specific peptidase 18 (USP18; also called UBP43) is a human-specific enzyme that removes ISG15 from conjugated proteins (Malakhov et al., 2002; Basters et al., 2017; Basters et al., 2018; Mustachio et al., 2018). Using ISG15 as bait, we obtained more than 300 candidate ISG15 substrates using immunoprecipitation-mass spectrometry. At present, more than 100 proteins have been established as substrates of ISG15, including p53, nuclear factor kappa B (NF-кB), KRAS, cyclin D, PTEN protein, STAT1, and retinoic acid-induced gene I (RIG-I) (Feng et al., 2008; Kim et al., 2008; Huang et al., 2014; Ganesan et al., 2016; Park et al., 2016; Mustachio et al., 2018). In this review, we discuss how ISG15 regulates viral replication, inflammation, cell proliferation and differentiation, and tumor genesis and development by modifying these proteins.

Currently, there are still many controversies regarding whether ISG15 exerts a tumor-suppressing effect or a cancerpromoting effect. Both unconjugated and conjugated ISG15 have demonstrated tumor-suppressing and cancer-promoting functions. Research results show that the tumor-suppressing function of unconjugated ISG15 is mainly related to its immune regulatory function (Desai, 2015). Yeung TL using laser microdissection and sequencing analysis that free ISG15 was highly expressed in serous ovarian cancer with high infiltration of $\mathrm{CD}^{+} \mathrm{T}$ cells (Yeung et al., 2018). In vitro experiments indicated that free ISG15 can increase the ISG modification of extracellular signal-regulated protein kinase one and the viability of natural killer $(\mathrm{NK})$ cells and $\mathrm{CD} 8^{+}$ T cells and enhance immune surveillance (Burks et al., 2015). Moreover, studies have shown that unconjugated ISG15 exerts a cancer-promoting function by enhancing the stem transformation and proliferation of tumor cells (Sainz et al., 2014; Chen et al., 2016). The same effect occurs in conjugated ISG15, which exerts a cancer-promoting effect by interacting with carcinogens (Burks et al., 2014), and a tumor-suppressing effect by regulating the function of p53 (Park et al., 2016; Jeon et al., 2017). Therefore, ISG15 can perform distinct functions depending on the cell type and physiological state, substrate, and subcellular location.

\section{ISG15 AND INNATE IMMUNITY}

Studies have shown that fibroblasts, monocytes, lymphocytes, neutrophils, plasma cells, and NK cells secrete small amounts of ISG15 under physiological conditions (Bogunovic et al., 2012). In addition, the expression of ISG15 can be affected by many factors. Viral and bacterial infections, LPS, DNA damage and other pathogenic stimuli can activate the expression of ISG15 (Malakhova et al., 2002; Pitha-Rowe et al., 2004). The free form of ISG15 binds to the LFA1 receptor on the surface of NK cells and T lymphocytes, increasing the release of type I and II IFNs and activating natural and acquired immunity (Swaim et al., 


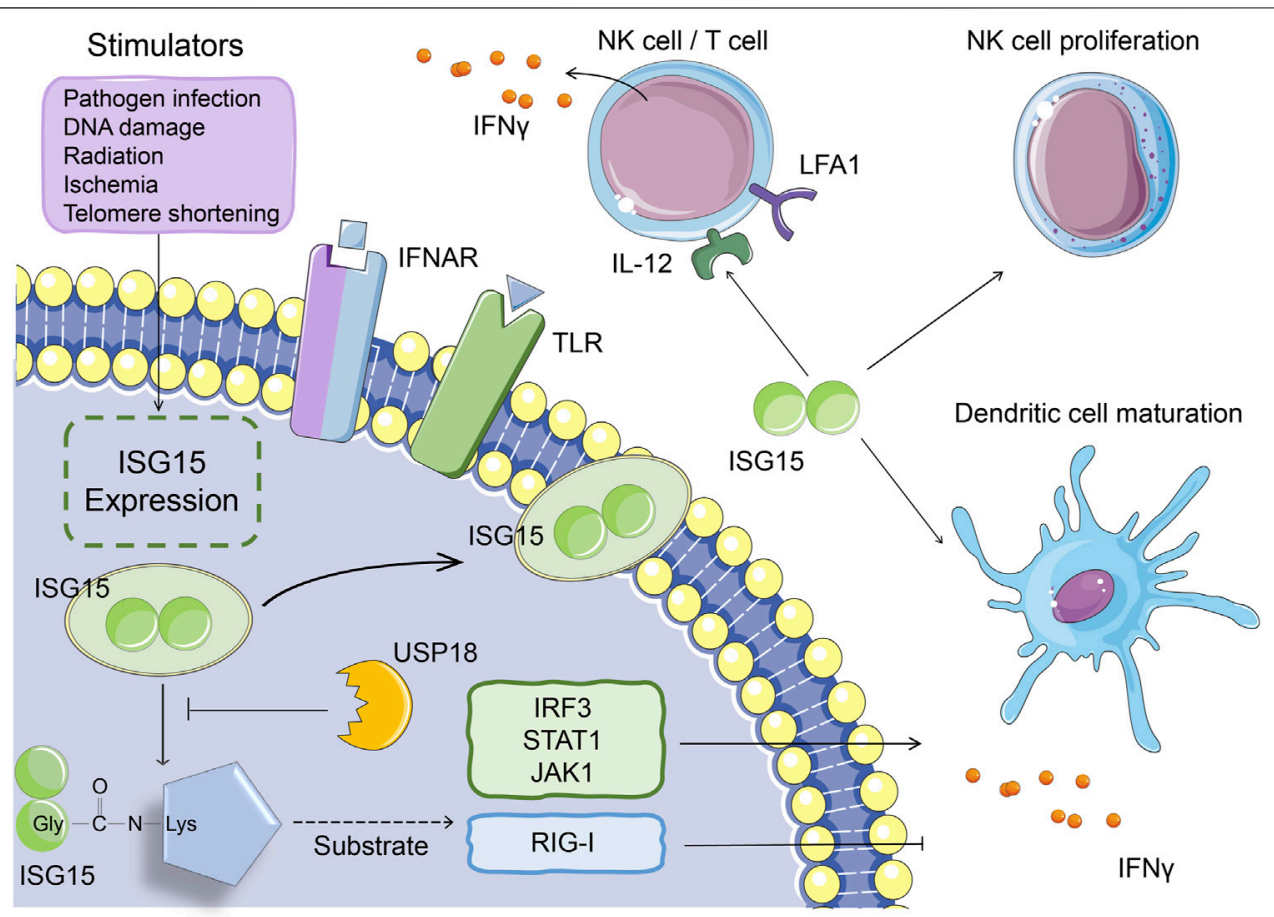

FIGURE 2 | The function of ISG15 in immune response. Under pathogenic stimuli such as viral and bacterial infections, LPS, and DNA damage, monocytes, lymphocytes, neutrophils, etc. can all secrete ISG15. Intracellular ISG15 can bind to proteins related to innate immune signaling pathways, activate IRF3, STAT1, JAK1 and other proteins, or inhibit protein activity (such as RIG-I), thereby promoting or inhibiting the secretion of IFN $\gamma$. The ISG15 secreted in vitro can bind to the LFA1 receptor on the cell surface, thereby promoting the secretion of IFN $\gamma$ from NK cells and T cells. It can also induce the proliferation of NK cells and the maturation of dendritic cells.

2017). ISG15 can also induce the proliferation of NK cells, the IFN $\gamma$ production of $\mathrm{NK}$ cells and $\mathrm{T}$ cells, the maturation of dendritic cells, and enhancement of antigen presentation and function as a chemokine that promotes the enrichment of neutrophils to inflammatory regions (Figure 2) (Morales et al., 2015; D’Cunha et al., 1996; Padovan et al., 2002; Owhashi et al., 2003; Recht et al., 1991).

Proteomic studies have identified that the immuneregulating factors interferon-regulated transcription factor 3 (IRF3), STAT1, and Janus kinase one function as substrates of ISG15 and that the ISGylation of these proteins increases the release of type I IFNs and ISGs, thereby extending the immune response signal cascade (Ganesan et al., 2016; Albert et al., 2018; Yoo et al., 2018; Malakhov et al., 2003). For example, when the host is infected, STAT1 ISGylation promotes the maintenance of phosphorylation and continuous activation of downstream signaling, which ultimately promotes a more powerful IFN response (Ganesan et al., 2016). In addition to positive regulation, ISG15 negatively regulates type I IFN signaling at multiple levels, such as ISGylation of the RIG-I protein, which inhibits IFN expression (Figure 2) (Zhao et al., 2005; Kim et al., 2008; Zhu et al., 2014; Du et al., 2018). On the one hand, because the process of covalent binding of ISG15 to the target protein is reversible, this binding can be dissociated by the ubiquitinspecific protease USP18, which indirectly regulates IFN expression. On the other hand, the deubiquitinating enzyme
USP18 can also directly inhibit type I IFN receptor signaling, thereby suppressing the immune response (Arimoto et al., 2017). The non-covalent interactions of ISG15 and USP18 prevent the ubiquitination of USP18 by S-phase kinaseassociated protein two and stabilize the downregulation of the IFN signaling pathway by USP18 (Tokarz et al., 2004; Zhang et al., 2015).

These results suggest that ISG15 can regulate immune function from multiple perspectives, such as stimulating immune cell maturation, regulating cytokine release, and affecting IFN signaling. In recent years, many studies have explored the role of ISG15 in antiviral innate immunity, especially in the process of viral infection, and the role of ISGylation of host and viral target proteins in immune defense. In this review, we explore this topic in detail.

\section{ANTIVIRAL EFFECTS OF ISGYLATION ON HOST PROTEINS AND THEIR FUNCTIONS}

Although the ISG15 protein was discovered in 1979, its nature and function were not elucidated for many years, until researchers discovered that IFN-induced ISG15 and its covalent form were implicated as a central player in the process of viral infection. Gene knockout, overexpression, genetic deletion of each component in the ISG15 cascade 


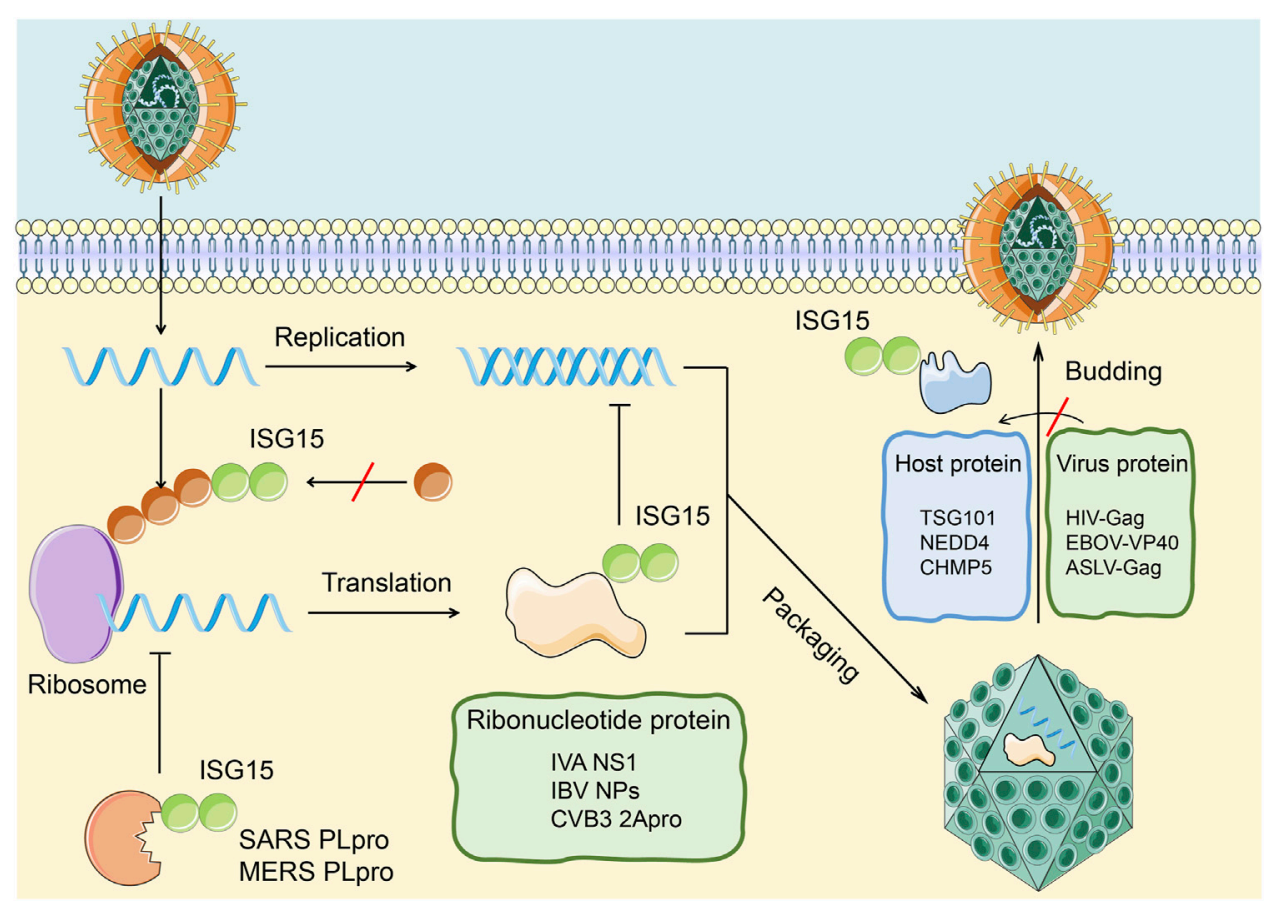

FIGURE 3 Antiviral effects of ISGylation on host and viral proteins. ISG15 affects the infection of cells by the virus through covalently binding with viral proteins and host proteins. 1. The combination of ISG15 and the viral nucleoprotein (green) can destroy the protein oligomerization and the ability of the viral nucleoprotein to inhibit virus replication. The ubiquitin-like modification formed by this combination can be cleaved by PLpro to restore the replication ability of the virus.2. The combination of ISG15 and host protein (blue) can inhibit the interaction between host protein and virus protein, thereby inhibiting the release of virus particles in the cell.

reaction process, and various other methods have since been used to determine whether ISG15 is involved in the host antiviral immune response (Campbell and Lenschow, 2013).

ISG15 can affect the antiviral immune response by binding to the target proteins of the IFN, NF- $\kappa \mathrm{B}$, and $\mathrm{c}$-Jun $\mathrm{N}$-terminal kinase (JNK) pathways (Jeon et al., 2009). Among them, the key factor for type I IFN response, IRF3, is a target of ISG15. The combination of ISG15 and IRF3 inhibited the proteasomal degradation of IRF3 and enhanced the intracellular IFN response (Ganesan et al., 2016). Concurrently, the covalent binding of ISG to the antiviral effector molecules K193, K360, and K366 can weaken the interaction between IRF3 and peptidylprolyl-cis-trans isomerase one and hinder the ubiquitination of IRF3 (Shi et al., 2010). Therefore, IRF3 can maintain its own activity after the modification of ISG and improve the IRF3mediated antiviral response by inhibiting its own degradation.

ISG15 can bind to protein kinase R (PKR), an IFN-inducible protein kinase activated by double-stranded RNA. Simultaneously ISG15 can also activate PKR in the absence of viral RNA. Activated PKR can inhibit protein translation by phosphorylation of eukaryotic initiation factor $2 \alpha$, and PKR activated by ISG15 can further promote IFN production (Okumura et al., 2013). In addition, RIG-I is the target protein of ISG15, and RIG-I can activate the RNA sensors of IRF3 and NF- $\kappa$ B. The covalent combination of ISG15 and RIG-I can downregulate signal transduction mediated by RIG-I. Free ISG15 can regulate the level of RIG-I by promoting the interaction between RIG-I and the autophagy substrate protein p62 (Nakashima et al., 2015; Du et al., 2018). ISGylation of phosphorylated STAT1 can also maintain its activity by inhibiting its own polyubiquitination and proteasomal degradation (Ganesan et al., 2016). In another example, ISGylation of filamin $B$ can negatively regulate IFNa-mediated c-Jun $\mathrm{N}$-terminal kinase signals and inhibit cell apoptosis (Jeon et al., 2009). ISG15 can also bind to ubiquitinconjugating enzyme 13 to inhibit the ubiquitination of transforming growth factor kinase one and negatively regulates the NF- $\kappa$ B pathway (Takeuchi and Yokosawa, 2005).

On the one hand, ISG15 influences antiviral immunity by ISGylation of host cell proteins and the relevant immune signaling pathways. On the other hand, ISG15 can affect virus replication, release, and latency in the host body through the ubiquitin-like modification of the virus protein to achieve antiviral immunity (Figure 3). Relevant examples are described in detail below.

\section{ISGYLATION OF VIRAL PROTEINS AND THEIR FUNCTIONS}

Lenschow and Werneke's team demonstrated that ISG15knockout mice were more susceptible to IAV and IBV, herpes simplex virus, norovirus, chikungunya virus, and other pathogens than wild-type mice (Lenschow et al., 2007; Werneke et al., 2011; Morales and Lenschow, 2013; Rodriguez et al., 2014). They demonstrated that both free and binding ISG15 expression is 
upregulated after pathogen infection, and both forms of ISG15 exhibit antiviral activity. For example, after IAV infection, free ISG15 can bind to the NS1 protein with seven lysine residues, which are potential target sites for ISGylation, blocking the nuclear localization of the NS1 protein and inhibiting virus replication, RNA processing (Jumat et al., 2016). At the same time, the ISGylation of NS1 can inhibit the interaction with PKR, which relieve the inhibition of NS1 protein on innate immunity and restoring IFN-induced anti-IAV activity (Pincetic et al., 2010).

As for IBV, nucleoprotein and matrix protein $\mathrm{M} 1$ are also targets for covalent binding of ISG15. Nucleoprotein ISGylation hinders the oligomerization of a large number of other nonconjugated nucleoproteins, inhibits the formation of IBV ribonucleic acid protein, and reduces viral protein synthesis and viral replication (Durfee et al., 2010; Zhao et al., 2016). Rahnefeld et al. found that the coxsackie virus CVB3 2A protease ISGylation can inhibit the cleavage of eukaryotic translation initiation factor $4 \mathrm{G}$ and reduce CVB3 replication (Rahnefeld et al., 2014).

Studies have shown that ISG15 can affect the release of HIV, Ebola virus, and avian sarcoma leukosis virus through different mechanisms. Pincetic and Okumura demonstrated that ISG15 inhibited the monoubiquitination of the HIV group-specific antigen protein, blocked its interaction with host tumor susceptibility gene 101, and inhibited the emergence and release of HIV (Okumura et al., 2006). When infected with Ebola, the ubiquitin ligase NEDD4 catalyzes the ubiquitination of the viral matrix protein VP40 and promotes the release of virus-like particles (Okumura et al., 2008). Lenschow and Malakhova demonstrated that ISG15 inhibits the transfer of ubiquitin-binding enzyme to NEDD4 and activity of NEDD4 ubiquitin-binding enzyme, thus inhibiting the budding and release of the Ebola virus (Malakhova and Zhang, 2008). ISGylation of charged multivesicular body protein 5 (CHMP5), a component of the endosome sorting complex, promotes its aggregation and the isolation of Vps4 coenzyme factor LIP5 and limits the membrane recruitment of Vps4 and its interaction with the avian sarcoma leukosis virus budding complex, thereby inhibiting the release of intracellular virus-like particles (Pincetic et al., 2010). In addition, researchers found that ISG15 can also affect the budding process of vesicular stomatitis virus by inhibiting the activity of NEDD4 and that ISG15 overexpression can significantly reduce the viral titer of its wild-type strains (Malakhova and Zhang, 2008).

Another study showed that ISG15 regulated the incubation period of the virus. Dai et al. used Illumina microarray technology to analyze the gene expression changes in primary human oral fibroblasts after infection with Kaposi's sarcoma-associated herpes virus and found that a series of IFN-stimulated genes were upregulated, especially ISG15 and ISG20, which maintain the virus incubation period by regulating Kaposi's sarcomaassociated herpes virus-specific microRNA (Dai et al., 2016). This reduces the expression of ISG15 during the incubation period of Kaposi's sarcoma-associated herpes virus infection and increases the expression of virus cleavage genes and the release of virus particles.
These results suggest that free or bound ISG15 produced by stress can regulate the function of viral proteins, inhibit viral replication, budding, and release. Thus, ISG15 may play a key role in inhibiting viral infection (Table 1).

\section{ISG15 PARTICIPATES IN NON-VIRAL INNATE IMMUNE RESPONSES}

Recent work has also highlighted the function of ISG15 in nonviral innate immune responses, such as pathogen defense responses, host damage and repair responses, and other host signaling pathways. ISG $15^{-/-}$mice are more susceptible to mycobacterium than wild-type mice, verifying that the degree of mycobacterium drop is not a determinant of susceptibility enhancement. Significantly increased cytokine release was detected in ISG $15^{-/-}$mice, and the cytokine storm induced by ISG15 knockout was blocked by tumor necrosis factor- $\alpha$-specific antibodies (Bogunovic et al., 2012; Kimmey et al., 2017). During Listeria monocytogenes infection, the expression of ISG15 increases, which depends on the cytosolic DNA-sensing pathway, and enhanced secretion of IL-6 and IL-8 was detected in ISG15-overexpressing cells (Radoshevich et al., 2015). These studies demonstrate that ISG15 plays an antagonistic role in the host response to pathogens and regulates cytokine signal transduction. Exogenous stimuli, such as DNA damage, radiation, ischemia, and telomere shortening, can also induce immune cells to produce ISG15 (Liu et al., 2004).

\section{SARS-COV-2 PAPAIN-LIKE PROTEASE: A DECONJUGATING PROTEASE}

The SARS-CoV-coronavirus genome encodes two viral proteases: PLpro and 3C-like protease. The structure and function of PLpro has been a hot topic in the molecular biology of coronavirus recently. PLpro is involved in cutting the N-terminal part of the SARS-CoV replicase polymerin and is a regulatory protein molecule for the formation of the SARS-CoV replicase complex (Shin et al., 2020). Results showed that SARS$\mathrm{CoV}$ PLpro protease is a virus-encoded DUB, which has an obvious deubiquitinating effect on cellular proteins (Klemm et al., 2020). PLpro is also active against ubiquitin and ISG15, which can negatively regulate the innate immune response to the virus (Shin et al., 2020). There are also OTU domain-containing proteases that can be encoded by Crimean-Congo hemorrhagic fever orthonairovirus, porcine reproductive and respiratory syndrome virus, and equine arteritis virus, which have properties similar to those of PLpro (Frias-Staheli et al., 2007). These proteins have been shown to reduce ubiquitin and ISG15 conjugates in cells. However, the researchers have compared SARS-CoV-2-PLpro with similar enzymes of other coronaviruses (SARS-CoV-1 and MERS). It was found that the SARS-CoV-2-PLpro enzyme processes ubiquitin and ISG15 in a different way with SARS-CoV-1-PLpro (Rut et al., 2020).

Recently, Huang and Zhang have made progress in elucidating the complex structure of SARS-CoV-2 PLpro and antiviral drug 
TABLE1 | Interaction between ISG15 and viral proteins.

\begin{tabular}{|c|c|c|c|}
\hline Viral proteins & Biological effects after ISGylation & Impact on viral infection & Reference \\
\hline IVA NS1 & $\begin{array}{l}\text { ISG15 inhibits viral proteins nuclear translocation and restores } \\
\text { host antiviral responses }\end{array}$ & Inhibits IBV replication & $\begin{array}{l}\text { (Tang et al., 2010; Zhao } \\
\text { et al., 2010) }\end{array}$ \\
\hline IBV NPS & $\begin{array}{l}\text { ISGylation of NPs inhibit the oligomerization of unmodified NPs, } \\
\text { which impedes viral RNA synthesis }\end{array}$ & Inhibits IBV replication & Zhao et al. (2016) \\
\hline CVB3 2Apro & $\begin{array}{l}\text { ISG15 inhibits its protease activity to restore host protein } \\
\text { translation }\end{array}$ & Inhibits CVB3 replication & Rahnefeld et al. (2014) \\
\hline HIV Gag & $\begin{array}{l}\text { ISG15 inhibits the monoubiquitination of Gag protein and block } \\
\text { its interaction with TSG101 }\end{array}$ & Inhibits the emergence and release of HIV. & Okumura et al. (2006) \\
\hline EBOV VP40 & $\begin{array}{l}\text { ISGylation of NEDD4 ubiquitin-binding enzyme inhibits its } \\
\text { interaction with VP40 }\end{array}$ & Inhibits the budding and release of Ebola virus & $\begin{array}{l}\text { (Yasuda et al., 2003; } \\
\text { Okumura et al., 2008) }\end{array}$ \\
\hline ASLV Gag & $\begin{array}{l}\text { The ISGylation of CHMP5 limits the membrane recruitment of } \\
\text { Vps4 and its interaction with the ASLV Gag }\end{array}$ & $\begin{array}{l}\text { Inhibits the ASLV budding complex, then inhibits the } \\
\text { release of intracellular virus-like particles }\end{array}$ & Pincetic et al. (2010) \\
\hline $\begin{array}{l}\text { SARS PLpro } \\
\text { MERS PLpro }\end{array}$ & $\begin{array}{l}\text { PLpro protease is a virus-encoded DUB, which active on } \\
\text { ubiquitin like molecule ISG15 }\end{array}$ & $\begin{array}{l}\text { Negatively regulates the innate immune response to } \\
\text { the virus }\end{array}$ & Rut et al. (2020) \\
\hline
\end{tabular}

discovery (Fu et al., 2021). They found that the small molecule inhibitor GRL0617 inhibited the activity of PLpro to shear the ubiquitin-like chain and the ubiquitin-like protein ISG15 chain in vitro and the ability to inhibit viral replication of SARS-CoV2. The structure of the inhibitor and protein complex and twodimensional NMR experiments revealed that GRL0617 interferes with protein-protein interaction between PLpro and ISG15, acting as an inhibitor for this interaction. They established that SARS-CoV2 protease PLpro is a target for antiviral drug development at the cellular and atomic resolution crystal structure levels and identified the binding site of GRL0617 as a hot spot for antiviral drug development targeting PLpro using a variety of biophysical methods.

\section{DISCUSSION}

ISG15 is a ubiquitin-like protein, produced by IFN, viruses, lipopolysaccharides, and other stimuli. ISG15 exerts antiviral effects by covalently binding to target proteins, inhibiting the release and replication of viral particles, and regulating the incubation period of viruses. In addition to the ISG15 covalent conjugate, the ISG15 monomer can promote the proliferation of NK cells and dendritic cells and enhance the chemotactic activity of neutrophils. Moreover, ISG15 is implicated in host damage, DNA repair, autophagy, protein translation, and other processes. ISG15 is also associated with the occurrence of cancer. However, there are still many unsolved mysteries about the biological function of ISG15 and the molecular mechanism underlying the antiviral effects of the ubiquitinlike modification system.

The PLP2 domains of many human and animal coronaviruses, such as the SARS coronavirus, MHV-A59, NL-63, and 229E, have demonstrated DUB activity, and the catalytic sequence of the PLP domain of these coronaviruses is highly conserved. However, it is still unclear whether DUB activity and regulation of the host natural immune response are the common characteristics of all PLpros, and the functional relationship between DUB activity of PLpro and its IFN antagonism needs further study.

In general, the diversity and broad spectrum of substrates, complexity of the ISG enzyme system, and cross-linking with the ubiquitination pathway all determine the complexity of ISG15 function. Further understanding of the molecular trajectory of the ubiquitin-like protein ISG15 may lead to new therapeutic strategies for antiviral treatment, immune function regulation, and cancer treatment.

\section{AUTHOR CONTRIBUTIONS}

$\mathrm{MZ}$ and JL conceived and drafted the manuscript. FZ, FW, HY, $\mathrm{JH}$, TL discussed the concepts of the manuscript. MZ drew the figures. FZ and LZ approved the version to be submitted. All authors contributed to the article and approved the submitted version.

\section{FUNDING}

This work was supported by a special program from the Chinese National Natural Science Funds (31871405 and 82041009 to FZ), Jiangsu National Science Foundation (BK20180043 and19KJA550003 to FZ).

\section{ACKNOWLEDGMENTS}

We would like to apologize to those researchers whose related work we were not able to cite in this review. 


\section{REFERENCES}

Albert, M., Bécares, M., Falqui, M., Fernández-Lozano, C., and Guerra, S. (2018). ISG15, a Small Molecule with Huge Implications: Regulation of Mitochondrial Homeostasis. Viruses 10. doi:10.3390/v10110629

Arimoto, K.-i., Löchte, S., Stoner, S. A., Burkart, C., Zhang, Y., Miyauchi, S., et al. (2017). STAT2 Is an Essential Adaptor in USP18-Mediated Suppression of Type I Interferon Signaling. Nat. Struct. Mol. Biol. 24, 279-289. doi:10.1038/nsmb.3378

Basters, A., Knobeloch, K. P., and Fritz, G. (2018). USP18 - a Multifunctional Component in the Interferon Response. Biosci. Rep. 38, BSR20180250. doi:10.1042/BSR20180250

Basters, A., Geurink, P. P., Röcker, A., Witting, K. F., Tadayon, R., Hess, S., et al. (2017). Structural Basis of the Specificity of USP18 toward ISG15. Nat. Struct. Mol. Biol. 24, 270-278. doi:10.1038/nsmb.3371

Bogunovic, D., Byun, M., Durfee, L. A., Abhyankar, A., Sanal, O., Mansouri, D., et al. (2012). Mycobacterial Disease and Impaired IFN- $\gamma$ Immunity in Humans with Inherited ISG15 Deficiency. Science 337, 1684-1688. doi:10.1126/ science. 1224026

Burks, J., Reed, R. E., and Desai, S. D. (2015). Free ISG15 Triggers an Antitumor Immune Response against Breast Cancer: a New Perspective. Oncotarget 6, 7221-7231. doi:10.18632/oncotarget.3372

Burks, J., Reed, R. E., and Desai, S. D. (2014). ISGylation Governs the Oncogenic Function of Ki-Ras in Breast Cancer. Oncogene 33, 794-803. doi:10.1038/ onc. 2012.633

Campbell, J. A., and Lenschow, D. J. (2013). Emerging Roles for Immunomodulatory Functions of Free ISG15. J. Interferon Cytokine Res. 33, 728-738. doi:10.1089/jir.2013.0064

Chen, R.-H., Du, Y., Han, P., Wang, H.-B., Liang, F.-Y., Feng, G.-K., et al. (2016). ISG15 Predicts Poor Prognosis and Promotes Cancer Stem Cell Phenotype in Nasopharyngeal Carcinoma. Oncotarget 7, 16910-16922. doi:10.18632/ oncotarget.7626

D’Cunha, J., Knight, E., Jr., Haas, A. L., Truitt, R. L., and Borden, E. C. (1996). Immunoregulatory Properties of ISG15, an Interferon-Induced Cytokine. Proc. Natl. Acad. Sci. 93, 211-215. doi:10.1073/pnas.93.1.211

Dai, L., Bai, L., Lin, Z., Qiao, J., Yang, L., Flemington, E. K., et al. (2016). Transcriptomic Analysis of KSHV-Infected Primary Oral Fibroblasts: The Role of Interferon-Induced Genes in the Latency of Oncogenic Virus. Oncotarget 7, 47052-47060. doi:10.18632/oncotarget.9720

Desai, S. D. (2015). ISG15: A Double Edged Sword in Cancer. Oncoimmunology 4, e1052935. doi:10.1080/2162402x.2015.1052935

Dos Santos, P. F., and Mansur, D. S. (2017). Beyond ISGlylation: Functions of Free Intracellular and Extracellular ISG15. J. Interferon Cytokine Res. 37, 246-253. doi:10.1089/jir.2016.0103

Du, Y., Duan, T., Feng, Y., Liu, Q., Lin, M., Cui, J., et al. (2018). LRRC25 Inhibits Type I IFN Signaling by Targeting ISG15-associated RIG-I for Autophagic Degradation. EMBO J. 37, 351-366. doi:10.15252/embj.201796781

Durfee, L. A., Lyon, N., Seo, K., and Huibregtse, J. M. (2010). The ISG15 Conjugation System Broadly Targets Newly Synthesized Proteins: Implications for the Antiviral Function of ISG15. Mol. Cel 38, 722-732. doi:10.1016/j.molcel.2010.05.002

Feng, Q., Sekula, D., Guo, Y., Liu, X., Black, C. C., Galimberti, F., et al. (2008). UBE1L Causes Lung Cancer Growth Suppression by Targeting Cyclin D1. Mol. Cancer Ther. 7, 3780-3788. doi:10.1158/1535-7163.mct-08-0753

Frias-Staheli, N., Giannakopoulos, N. V., Kikkert, M., Taylor, S. L., Bridgen, A., Paragas, J., et al. (2007). Ovarian Tumor Domain-Containing Viral Proteases Evade Ubiquitin- and ISG15-dependent Innate Immune Responses. Cell Host \& Microbe 2, 404-416. doi:10.1016/j.chom.2007.09.014

Fu, Z., Huang, B., Tang, J., Liu, S., Liu, M., Ye, Y., et al. (2021). The Complex Structure of GRL0617 and SARS-CoV-2 PLpro Reveals a Hot Spot for Antiviral Drug Discovery. Nat. Commun. 12, 488. doi:10.1038/s41467-020-20718-8

Ganesan, M., Poluektova, L. Y., Tuma, D. J., Kharbanda, K. K., and Osna, N. A. (2016). Acetaldehyde Disrupts Interferon Alpha Signaling in Hepatitis C VirusInfected Liver Cells by Up-Regulating USP18. Alcohol. Clin. Exp. Res. 40, 2329-2338. doi:10.1111/acer.13226

Hermann, M., and Bogunovic, D. (2017). ISG15: In Sickness and in Health. Trends Immunol. 38, 79-93. doi:10.1016/j.it.2016.11.001
Huang, Y. F., Wee, S., Gunaratne, J., Lane, D. P., and Bulavin, D. V. (2014). Isg15 Controls P53 Stability and Functions. Cell Cycle 13, 2200-2210. doi:10.4161/ cc. 29209

Jeon, Y. J., Choi, J. S., Lee, J. Y., Yu, K. R., Kim, S. M., Ka, S. H., et al. (2009). ISG15 Modification of Filamin B Negatively Regulates the Type I Interferon-induced JNK Signalling Pathway. EMBO Rep. 10, 374-380. doi:10.1038/embor.2009.23

Jeon, Y. J., Park, J. H., and Chung, C. H. (2017). Interferon-Stimulated Gene 15 in the Control of Cellular Responses to Genotoxic Stress. Mol. Cell 40, 83-89. doi:10.14348/molcells.2017.0027

Jumat, M. R., Wong, P., Lee, R. T. C., Maurer-Stroh, S., Tan, B. H., and Sugrue, R. J. (2016). Molecular and Biochemical Characterization of the NS1 Protein of Non-cultured Influenza B Virus Strains Circulating in Singapore. Microb. Genom 2, e000082. doi:10.1099/mgen.0.000082

Kim, M.-J., Hwang, S.-Y., Imaizumi, T., and Yoo, J.-Y. (2008). Negative Feedback Regulation of RIG-I-Mediated Antiviral Signaling by Interferon-Induced ISG15 Conjugation. J. Virol. 82, 1474-1483. doi:10.1128/jvi.01650-07

Kimmey, J. M., Campbell, J. A., Weiss, L. A., Monte, K. J., Lenschow, D. J., and Stallings, C. L. (2017). The Impact of ISGylation during Mycobacterium tuberculosis Infection in Mice. Microbes Infect. 19, 249-258. doi:10.1016/ j.micinf.2016.12.006

Klemm, T., Ebert, G., Calleja, D. J., Allison, C. C., Richardson, L. W., Bernardini, J. P., et al. (2020). Mechanism and Inhibition of the Papain-like Protease, PLpro, of SARS-CoV-2. EMBO J. 39, e106275. doi:10.15252/embj.2020106275

Langevin, C., van der Aa, L. M., Houel, A., Torhy, C., Briolat, V., Lunazzi, A., et al. (2013). Zebrafish ISG15 Exerts a strong Antiviral Activity against RNA and DNA Viruses and Regulates the Interferon Response. J. Virol. 87, 10025-10036. doi:10.1128/jvi.01294-12

Lenschow, D. J., Lai, C., Frias-Staheli, N., Giannakopoulos, N. V., Lutz, A., Wolff, T., et al. (2007). From the Cover: IFN-Stimulated Gene 15 Functions as a Critical Antiviral Molecule against Influenza, Herpes, and Sindbis Viruses. Proc. Natl. Acad. Sci. 104, 1371-1376. doi:10.1073/pnas.0607038104

Liu, M., Hummer, B. T., Li, X., and Hassel, B. A. (2004). Camptothecin Induces the Ubiquitin-like Protein, ISG15, and Enhances ISG15 Conjugation in Response to Interferon. J. Interferon Cytokine Res. 24, 647-654. doi:10.1089/jir.2004.24.647

Loeb, K. R., and Haas, A. L. (1992). The Interferon-Inducible 15-kDa Ubiquitin Homolog Conjugates to Intracellular Proteins. J. Biol. Chem. 267, 7806-7813. doi:10.1016/s0021-9258(18)42585-9

Malakhov, M. P., Kim, K. I., Malakhova, O. A., Jacobs, B. S., Borden, E. C., and Zhang, D.-E. (2003). High-throughput Immunoblotting. J. Biol. Chem. 278, 16608-16613. doi:10.1074/jbc.m208435200

Malakhov, M. P., Malakhova, O. A., Kim, K. I., Ritchie, K. J., and Zhang, D.-E. (2002). UBP43 (USP18) Specifically Removes ISG15 from Conjugated Proteins. J. Biol. Chem. 277, 9976-9981. doi:10.1074/jbc.m109078200

Malakhova, O. A., and Zhang, D.-E. (2008). ISG15 Inhibits Nedd4 Ubiquitin E3 Activity and Enhances the Innate Antiviral Response*. J. Biol. Chem. 283, 8783-8787. doi:10.1074/jbc.c800030200

Malakhova, O., Malakhov, M., Hetherington, C., and Zhang, D.-E. (2002). Lipopolysaccharide Activates the Expression of ISG15-specific Protease UBP43 via Interferon Regulatory Factor 3. J. Biol. Chem. 277, 14703-14711. doi:10.1074/jbc.m111527200

Morales, D. J., and Lenschow, D. J. (2013). The Antiviral Activities of ISG15. J. Mol. Biol. 425, 4995-5008. doi:10.1016/j.jmb.2013.09.041

Morales, D. J., Monte, K., Sun, L., Struckhoff, J. J., Agapov, E., Holtzman, M. J., et al. (2015). Novel Mode of ISG15-Mediated protection against Influenza A Virus and Sendai Virus in Mice. J. Virol. 89, 337-349. doi:10.1128/jvi.02110-14

Mustachio, L. M., Lu, Y., Kawakami, M., Roszik, J., Freemantle, S. J., Liu, X., et al. (2018). Evidence for the ISG15-specific Deubiquitinase USP18 as an Antineoplastic Target. Cancer Res. 78, 587-592. doi:10.1158/0008-5472.can17-1752

Nakashima, H., Nguyen, T., Goins, W. F., and Chiocca, E. A. (2015). Interferonstimulated Gene 15 (ISG15) and ISG15-Linked Proteins Can Associate with Members of the Selective Autophagic Process, Histone Deacetylase 6 (HDAC6) and SQSTM1/p62. J. Biol. Chem. 290, 1485-1495. doi:10.1074/ jbc.m114.593871

Okumura, A., Lu, G., Pitha-Rowe, I., and Pitha, P. M. (2006). Innate Antiviral Response Targets HIV-1 Release by the Induction of Ubiquitin-like Protein ISG15. Proc. Natl. Acad. Sci. 103, 1440-1445. doi:10.1073/pnas.0510518103 
Okumura, A., Pitha, P. M., and Harty, R. N. (2008). ISG15 Inhibits Ebola VP40 VLP Budding in an L-domain-dependent Manner by Blocking Nedd4 Ligase Activity. Proc. Natl. Acad. Sci. 105, 3974-3979. doi:10.1073/pnas.0710629105

Okumura, F., Okumura, A. J., Uematsu, K., Hatakeyama, S., Zhang, D.-E., and Kamura, T. (2013). Activation of Double-Stranded RNA-Activated Protein Kinase (PKR) by Interferon-Stimulated Gene 15 (ISG15) Modification Down-Regulates Protein Translation. J. Biol. Chem. 288, 2839-2847. doi:10.1074/jbc.m112.401851

Owhashi, M., Taoka, Y., Ishii, K., Nakazawa, S., Uemura, H., and Kambara, H. (2003). Identification of a Ubiquitin Family Protein as a Novel Neutrophil Chemotactic Factor. Biochem. Biophysical Res. Commun. 309, 533-539. doi:10.1016/j.bbrc.2003.08.038

Padovan, E., Terracciano, L., Certa, U., Jacobs, B., Reschner, A., Bolli, M., et al. (2002). Interferon Stimulated Gene 15 Constitutively Produced by Melanoma Cells Induces E-Cadherin Expression on Human Dendritic Cells. Cancer Res. 62, 3453-3458. doi:10.1111/omi.12270/v1/review2

Park, J. H., Yang, S. W., Park, J. M., Ka, S. H., Kim, J.-H., Kong, Y.-Y., et al. (2016). Positive Feedback Regulation of P53 Transactivity by DNA Damage-Induced ISG15 Modification. Nat. Commun. 7, 12513. doi:10.1038/ncomms12513

Pincetic, A., Kuang, Z., Seo, E. J., and Leis, J. (2010). The Interferon-Induced Gene ISG15 Blocks Retrovirus Release from Cells Late in the Budding Process. J. Virol. 84, 4725-4736. doi:10.1128/jvi.02478-09

Pitha-Rowe, I., Hassel, B. A., and Dmitrovsky, E. (2004). Involvement of UBE1L in ISG15 Conjugation during Retinoid-Induced Differentiation of Acute Promyelocytic Leukemia. J. Biol. Chem. 279, 18178-18187. doi:10.1074/jbc.m309259200

Potter, J. L., Narasimhan, J., Mende-Mueller, L., and Haas, A. L. (1999). Precursor Processing of Pro-ISG15/UCRP, an Interferon- $\beta$-Induced Ubiquitin-like Protein. J. Biol. Chem. 274, 25061-25068. doi:10.1074/jbc.274.35.25061

Radoshevich, L., Impens, F., Ribet, D., Quereda, J. J., Nam Tham, T., Nahori, M. A., et al. (2015). ISG15 Counteracts Listeria Monocytogenes Infection. Elife 4, e06848. doi:10.7554/eLife.06848

Rahnefeld, A., Klingel, K., Schuermann, A., Diny, N. L., Althof, N., Lindner, A., et al. (2014). Ubiquitin-like Protein ISG15 (Interferon-stimulated Gene of 15 $\mathrm{kDa}$ ) in Host Defense against Heart Failure in a Mouse Model of Virus-Induced Cardiomyopathy. Circulation 130, 1589-1600. doi:10.1161/circulationaha.114.009847

Recht, M., Borden, E. C., and Knight, E., Jr. (1991). A Human 15-kDa IFN-Induced Protein Induces the Secretion of IFN-Gamma. J. Immunol. 147, 2617-2623. doi:10.1111/j.1365-2249.1990.tb05403.x

Rodriguez, M. R., Monte, K., Thackray, L. B., and Lenschow, D. J. (2014). ISG15 Functions as an Interferon-Mediated Antiviral Effector Early in the Murine Norovirus Life Cycle. J. Virol. 88, 9277-9286. doi:10.1128/jvi.01422-14

Rut, W., Lv, Z., Zmudzinski, M., Patchett, S., Nayak, D., Snipas, S. J., et al. (2020). Olsen, Activity Profiling and Crystal Structures of Inhibitor-Bound SARS-CoV-2 PapainLike Protease: A Framework for Anti-COVID-19 Drug Design. Sci. Adv. 6.

Sainz, B., Jr., Martín, B., Tatari, M., Heeschen, C., and Guerra, S. (2014). ISG15 Is a Critical Microenvironmental Factor for Pancreatic Cancer Stem Cells. Cancer Res. 74, 7309-7320. doi:10.1158/0008-5472.can-14-1354

Shi, H.-X., Yang, K., Liu, X., Liu, X.-Y., Wei, B., Shan, Y.-F., et al. (2010). Positive Regulation of Interferon Regulatory Factor 3 Activation by Herc5 via ISG15 Modification. Mol. Cel Biol 30, 2424-2436. doi:10.1128/mcb.01466-09

Shin, D., Mukherjee, R., Grewe, D., Bojkova, D., Baek, K., Bhattacharya, A., et al. (2020). Papain-like Protease Regulates SARS-CoV-2 Viral Spread and Innate Immunity. Nature 587, 657-662. doi:10.1038/s41586-020-2601-5

Sooryanarain, H., Rogers, A. J., Cao, D., Haac, M. E. R., Karpe, Y. A., and Meng, X. J. (2017). ISG15 Modulates Type I Interferon Signaling and the Antiviral Response during Hepatitis E Virus Replication. J. Virol. 91, e00621. doi:10.1128/JVI.00621-17

Swaim, C. D., Scott, A. F., Canadeo, L. A., and Huibregtse, J. M. (2017). Extracellular ISG15 Signals Cytokine Secretion through the LFA-1 Integrin Receptor. Mol. Cel 68, 581-590. e585. doi:10.1016/j.molcel.2017.10.003

Takeuchi, T., Koinuma, S., Yokosawa, H., and Arata, Y. (2019). An Approach for the Identification of Proteins Modified with ISG15. Methods Mol. Biol. 1934, 235-246. doi:10.1007/978-1-4939-9055-9_15

Takeuchi, T., and Yokosawa, H. (2005). ISG15 Modification of Ubc13 Suppresses its Ubiquitin-Conjugating Activity. Biochem. Biophysical Res. Commun. 336, 9-13. doi:10.1016/j.bbrc.2005.08.034
Tang, Y., Zhong, G., Zhu, L., Liu, X., Shan, Y., Feng, H., et al. (2010). Herc5 Attenuates Influenza A Virus by Catalyzing ISGylation of Viral NS1 Protein. J. Immunol. 184, 5777-5790. doi:10.4049/jimmunol.0903588

Tokarz, S., Berset, C., La Rue, J., Friedman, K., Nakayama, K.-I., Nakayama, K., et al. (2004). The ISG15 Isopeptidase UBP43 Is Regulated by Proteolysis via the SCFSkp2 Ubiquitin Ligase. J. Biol. Chem. 279, 46424-46430. doi:10.1074/jbc.m403189200

Werneke, S. W., Schilte, C., Rohatgi, A., Monte, K. J., Michault, A., ArenzanaSeisdedos, F., et al. (2011). ISG15 Is Critical in the Control of Chikungunya Virus Infection Independent of UbE1L Mediated Conjugation. Plos Pathog. 7, e1002322. doi:10.1371/journal.ppat.1002322

Yasuda, J., Nakao, M., Kawaoka, Y., and Shida, H. (2003). Nedd4 Regulates Egress of Ebola Virus-like Particles from Host Cells. J. Virol. 77, 9987-9992. doi:10.1128/jvi.77.18.9987-9992.2003

Yeung, T. L., Tsai, C. C., Leung, C. S., Au Yeung, C. L., Thompson, M. S., Lu, K. H., et al. (2018). ISG15 Promotes ERK1 ISGylation, CD8+ T Cell Activation and Suppresses Ovarian Cancer Progression. Cancers (Basel) 10, 464. doi:10.3390/ cancers 10120464

Yoo, L., Yoon, A.-R., Yun, C.-O., and Chung, K. C. (2018). Covalent ISG15 Conjugation to CHIP Promotes its Ubiquitin E3 Ligase Activity and Inhibits Lung Cancer Cell Growth in Response to Type I Interferon. Cell Death Dis 9, 97. doi:10.1038/s41419-017-0138-9

Yuan, W., and Krug, R. M. (2001). Influenza B Virus NS1 Protein Inhibits Conjugation of the Interferon (IFN)-induced Ubiquitin-like ISG15 Protein. EMBO J. 20, 362-371. doi:10.1093/emboj/20.3.362

Zhang, X., Bogunovic, D., Payelle-Brogard, B., Francois-Newton, V., Speer, S. D., Yuan, C., et al. (2015). Human Intracellular ISG15 Prevents Interferon-A/ $\beta$ Over-amplification and Auto-Inflammation. Nature 517, 89-93. doi:10.1038/ nature 13801

Zhao, C., Denison, C., Huibregtse, J. M., Gygi, S., and Krug, R. M. (2005). Human ISG15 Conjugation Targets Both IFN-Induced and Constitutively Expressed Proteins Functioning in Diverse Cellular Pathways. Proc. Natl. Acad. Sci. 102, 10200-10205. doi:10.1073/pnas.0504754102

Zhao, C., Hsiang, T.-Y., Kuo, R.-L., and Krug, R. M. (2010). ISG15 Conjugation System Targets the Viral NS1 Protein in Influenza A Virus-Infected Cells. Proc. Natl. Acad. Sci. USA 107, 2253-2258. doi:10.1073/ pnas.0909144107

Zhao, C., Sridharan, H., Chen, R., Baker, D. P., Wang, S., and Krug, R. M. (2016). Influenza B Virus Non-structural Protein 1 Counteracts ISG15 Antiviral Activity by Sequestering ISGylated Viral Proteins. Nat. Commun. 7, 12754. doi:10.1038/ncomms12754

Zhu, Z., Yang, Y., Wei, J., Shao, D., Shi, Z., Li, B., et al. (2014). Type I InterferonMediated Immune Response against Influenza A Virus Is Attenuated in the Absence of P53. Biochem. Biophysical Res. Commun. 454, 189-195. doi:10.1016/ j.bbrc.2014.10.067

Zuo, C., Sheng, X., Ma, M., Xia, M., and Ouyang, L. (2016). ISG15 in the Tumorigenesis and Treatment of Cancer: An Emerging Role in Malignancies of the Digestive System. Oncotarget 7, 74393-74409. doi:10.18632/oncotarget.11911

Conflict of Interest: The research was conducted in the absence of any commercial or financial relationships that could be construed as a potential conflict of interest.

Publisher's Note: All claims expressed in this article are solely those of the authors and do not necessarily represent those of their affiliated organizations, or those of the publisher, the editors and the reviewers. Any product that may be evaluated in this article, or claim that may be made by its manufacturer, is not guaranteed or endorsed by the publisher.

Copyright (C) 2021 Zhang, Li, Yan, Huang, Wang, Liu, Zeng and Zhou. This is an open-access article distributed under the terms of the Creative Commons Attribution License (CC BY). The use, distribution or reproduction in other forums is permitted, provided the original author(s) and the copyright owner(s) are credited and that the original publication in this journal is cited, in accordance with accepted academic practice. No use, distribution or reproduction is permitted which does not comply with these terms. 